\title{
Challenges Associated with Multi-institutional Multi-site Clinical Trial Collaborations: Lessons from a Diabetes Self-Management Interventions Study in Primary Care
}

\section{Samuel N Forjuoh ${ }^{1,2 *}$, Janet W Helduser ${ }^{3}$, Jane N Bolin ${ }^{3}$ and Marcia G Ory ${ }^{2}$}

${ }^{1}$ Department of Family and Community Medicine, Baylor Scott and White Health, College of Medicine, Texas A\&M Health Science Center, Temple, TX, USA ${ }^{2}$ Department of Health Promotion and Community Health Sciences, School of Public Health, Texas A\&M Health Science Center, College Station, TX, USA ${ }^{3}$ Department of Health Policy and Management, School of Public Health, Texas A\&M Health Science Center, College Station, TX, USA

\begin{abstract}
Purpose: Project management for multi-institutional, multi-site clinical trials poses significant challenges. We describe the response to challenges encountered in a 5-year National Institutes of Health multi-institutional 7-site randomized controlled trial of type 2 diabetes (T2DM) self-management interventions study.

Methods: The collaborating institutions consisted of a large 220,000-member integrated healthcare system and a university academic health science center partner. The clinical team comprised the principal investigator and research coordinators covering 6 of the 7 clinical sites, while the academic team comprised the co-principal investigator, coinvestigators, and other research and clinical coordination staff. Subjects recruited for the study had a glycosylated hemoglobin $\geq 7.5$ within the last 6 months and received primary care at the participating clinics. Patients who met the inclusion criteria were consented at private orientation meetings, randomized into one of 4 study arms, and followed every 6 months over a 24-month period for data collection.

Results: The encountered challenges concerned: 1) communication across the multiple clinic sites; 2) multiinstitutional coordinator training; 3) multiple record-keeping methods; 4) clinical access for academic personnel; 5) unanticipated clinical coordinator turnover; 6) subject recruitment and retention; and 7) multiple Institutional Review Boards (IRBs). Solutions included conducting full team weekly or bimonthly research meetings, coordinator crosstraining, adding study-specific templates with downloadable fields, developing a protocol for working with single point of contact in each clinic, securing commitment from the centralized clinical system to dedicate coordinator(s) to the project for the duration of the study period, setting explicit monthly recruitment goals for each clinic, and establishing a lead IRB up-front.

Conclusion: Our challenges reflect the complexity of clinical trial collaborations across clinical and academic partners. Of critical importance to the success of clinical/academic collaborations is the commitment by all institutions for advance determination of communication strategies, IRB processes, records access and storage systems, and online training needs.
\end{abstract}

Trial Registration Number/Site: NCT01221090, https://clinicaltrials.gov

Keywords: Research management; Clinical trial management; Research collaboration; Inter-institutional research; Type 2 diabetes

\section{Introduction}

Researchers conducting clinical trials in multiple clinical sites with targeted populations face significant management challenges to maintain data integrity, while obtaining and retaining an adequate sample. These challenges can be further compounded when multiple institutions are involved, such as an integrated healthcare system and an academic-based partner. For example, communication among project team members can present a challenge to management of multi-site collaborations. Richards described communication as "the force that allows groups to take on their own identity" [1]. Effective communication for a research team is generally thought to be a function of pre-planning, adaption to various leadership styles, and organizational commitment [2-4]. However, a multi-site clinical trial does not necessarily fit the traditional hierarchical organizational model $[3,5]$. Within each clinical site, between clinical sites, and between clinical and academic collaborators, there must be common management strategies in place. Multi-site and multi-institutional studies present the added communication challenges of travel distance and time.

Whether they are a clinic or academic employee, research coordinators, in particular, have significant responsibility for organizing and overseeing a site's participation in multi-site clinical trials. Few organizations have exactly the same training requirements for coordinators and, as the number of clinical trial sites increases, these variations can present challenges in trial management. Training variability has the potential to create discrepancies among study staff regarding operating procedures, data collection, and record-keeping. A high degree of control in protocol implementation, data collection, and reporting has been identified as a key component in successfully managing multi-institutional randomized controlled trials (RCTs) and can be achieved, in part, through standardized training requirements $[3,6]$.

*Corresponding author: Samuel N Forjuoh, Department of Family and Community Medicine, Texas A\&M Health Science Center College of Medicine, Baylor Scott and White Health, Santa Fe - Century Square Building, 1402 West Ave H, Texas 76504 USA, Tel: +1-254-771-7695; Fax: +1-254-771-8493; E-mail: sforjuoh@sw.org

Received March 20, 2015; Accepted April 20, 2015; Published April 22, 2015

Citation: Forjuoh SN, Helduser JW, Bolin JN, Ory MG (2015) Challenges Associated with Multi-institutional Multi-site Clinical Trial Collaborations: Lessons from a Diabetes Self-Management Interventions Study in Primary Care. J Clin Trials 5: 219. doi:10.4172/2167-0870.1000219

Copyright: (c 2015 Forjuoh SN, et al. This is an open-access article distributed under the terms of the Creative Commons Attribution License, which permits unrestricted use, distribution, and reproduction in any medium, provided the original author and source are credited. 
Although variations exist in training programs for research staff, the most significant factor affecting the way clinical data are collected is the Health Insurance Portability and Accountability Act (HIPAA). Designed to protect the privacy and security of patients' personal health information (PHI), failure to comply with HIPAA regulations carries with it a risk for penalties and fines [7]. HIPAA's non-compliance associated penalties, including required compliance training, are two statutory mechanisms designed to limit access to electronic health records (EHR) [8,9]. In multi-institutional research studies, however, when employees of academic institutions are also responsible for scheduling, collecting, and recording patient data, HIPAA's statutorily designed limited access to EHR-based information presents a significant barrier.

In their 2010 study of methodological challenges to conducting a multi-site RCT of massage therapy in hospice, Kutner et al. reported an average turnover rate of $68 \%$ among study staff. Reasons for coordinator turnover included employees leaving the organization and competition for experienced coordinators, even within the home organization [4]. Turnover of key study staff can have a particularly devastating impact on multi-site studies, where standardized subject recruitment and retention plans are essential.

Recruitment and retention of subjects is challenging for all aspects of any research study [10-12]. Engaging study participants throughout the course of the study enhances retention, despite adding another layer of complexity to the coordinator role [13]. Regular communication between clinical trial participants and study coordinators to establish some level of trust may be necessary to enhance compliance and retention, however, engaging study participants throughout the course of a longitudinal RCT - and across multiple sites - is a challenge even for the most experienced coordinator.

A recent literature review on institutional review boards (IRBs) in the United States examined IRB structures, processes, outcomes, and variations. Inefficiencies were found to exist in multicenter reviews, as interpretations of federal regulations can be inconsistent between institutions [14]. Additional IRB-related challenges to multiinstitutional studies have ranged from non-standard submission formats and review criteria to delays in processing requests due to multiple IRB involvement [15-18].

We describe the strategies and responses to the challenges encountered in a 5-year National Institutes of Health (NIH) funded multi-institutional, 7-site randomized controlled trial of type 2 diabetes (T2DM) self-management interventions study in a primary care setting under the supervision of two IRBs.

\section{Methods}

The project was an NIH-funded study to examine the impact of selfmanagement interventions on clinical and self-care outcomes in adults with type 2 diabetes (T2DM). The interventions were: 1) participation in the 6 session Chronic Disease Self-management Program (CDSMP) developed by Stanford University [19]; 2) use of a Personal Digital Assistant (PDA) Tungsten E2 model; 3) participation in both the CDSMP and use of the PDA; and 4) control, i.e., usual care.

The two collaborating institutions included a large 220,000-member integrated healthcare system from which all subjects were recruited and an academic health science center partner. The clinical team comprised the principal investigator and research coordinators covering 6 of the 7 clinical sites, while the academic team comprised the co-principal investigator, co-investigators, and other research and clinical coordination staff. All subjects received primary care at one of the 7 participating clinics. One clinic, located in an urban setting, had a university-based employee as its research coordinator, while the other 6 clinics used coordinators employed by the healthcare system.

Adults with a glycosylated hemoglobin $(\mathrm{HbAlc}) \geq 7.5$ within the last 6 months and no reports of alcoholism or drug abuse during the same time period were considered as potential subjects. Seventy-four doctors at the 7 clinic locations assisted with subject recruitment by signing letters inviting participation to a list of their qualifying patients.

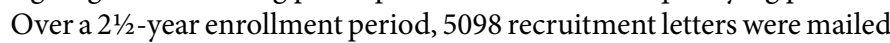
to qualifying patients. Other recruitment strategies included posters in the clinic lobbies and oral referrals by physicians and patient educators.

Research coordinators screened by telephone a total of 1897 patients. Three hundred and seventy-six patients who met all inclusion criteria, after being screened by telephone, were consented at private orientation meetings, enrolled, and randomized into one of the 4 arms of the study - educational intervention (CDSMP), technology intervention (PDA), both, or control - to determine the potential impact of these interventions on $\mathrm{HbA1c}$ in adults with T2DM. During the orientation visit, a baseline survey, vital signs, and clinical measurements were obtained. Follow-up visits to obtain updated vital signs and $\mathrm{HbAlc}$ laboratory values and to repeat the survey on health, physical activity, and diabetes self-care, were scheduled at $6,12,18$, and 24 months. Details of the study have been published elsewhere [20,21].

The project management plan that was initially proposed included having weekly face-to-face meetings of the co-Principal investigators and key personnel, bi-monthly conference calls with the full research team, frequent separate meetings among the coordinators, especially prior to the full research team conference call, and biannual faceto-face retreats. The twice monthly conference calls with the entire research team provided an opportunity to identify challenges, which were documented by the academic research team coordinator or clinical administrative assistant during the course of the project. Solutions were discussed in regular team meetings and typically required follow-up by coordinators from both institutions. This article represents a synthesis of major challenges and solutions identified and acted upon by the project team leadership.

\section{Results}

The specific management challenges encountered during our project are summarized in Table 1 . These challenges can be categorized into the following areas: 1) communication across multiple clinical sites and institutions, and between investigators; 2) variations in coordinator training requirements; 3) variations in record-keeping methods; 4) limited accessibility to facilities and EHR by key academic study personnel; 5) high clinical coordinator turnover; 6) subject recruitment and retention; and 7) use of multiple IRBs.

For example, we experienced an $86 \%$ turnover among the research coordinators, often due to re-assignments within the same healthcare system. This made consistent coverage at clinic sites especially challenging. Additionally, participant attrition was identified as a major challenge. $18 \%$ of the subjects withdrew completely prior to the 24-month study completion. A much larger percentage withdrew from active participation, but allowed continued access to their EHR for laboratory results (HbAlc) and vital signs. Our organizational responses and strategies for addressing the challenges experienced by our coordinators during this multi-site, multi-institutional study are presented in Table 2. 
Citation: Forjuoh SN, Helduser JW, Bolin JN, Ory MG (2015) Challenges Associated with Multi-institutional Multi-site Clinical Trial Collaborations: Lessons from a Diabetes Self-Management Interventions Study in Primary Care. J Clin Trials 5: 219. doi:10.4172/2167-0870.1000219

Table 1: Specific challenges encountered in our multi-site, multi-institutional clinical trial collaborations.

- Communication across multiple clinic sites separated by up to 100 miles

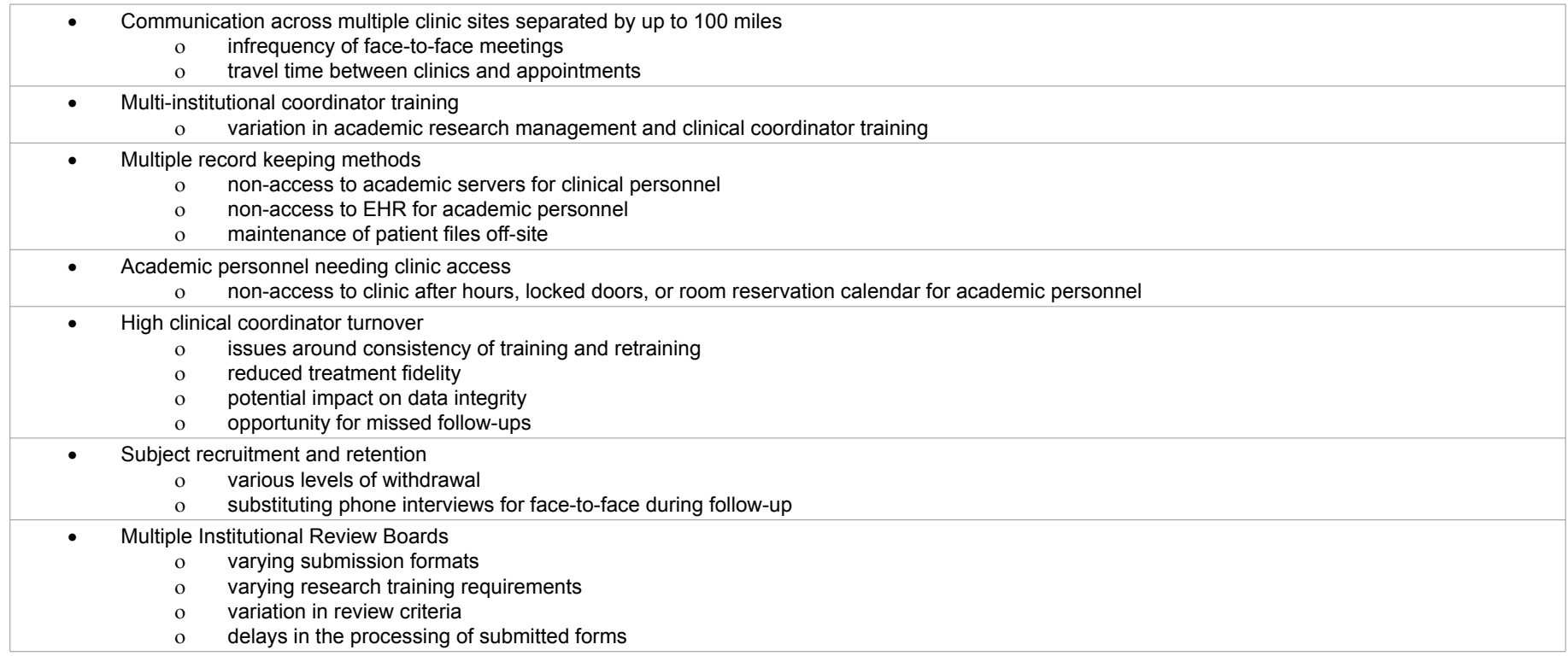

Table 2: Solution approaches used and recommended for improved management of clinical trial collaborations.

\begin{tabular}{|c|c|}
\hline Challenges & Recommended Solutions and Opportunities \\
\hline Communication across multiple clinic sites & $\begin{array}{l}\text { - Conducted weekly or bimonthly research meetings for the full team via phone. } \\
\text { - } \quad \text { Conducted separate coordinator meetings for clarification on minor recruitment issues ( } \geq 1 \text { representative per clinic site). }\end{array}$ \\
\hline Multi-institutional coordinator training & - Conducted coordinator cross-training \\
\hline Multiple record-keeping methods & $\begin{array}{l}\text { - } \quad \text { Added study-specific templates with downloadable fields to the EHR. } \\
\text { - } \quad \text { Provided uniform data collection forms (paper forms w/Access database or data entry directly into netbook/laptop). } \\
\text { - } \quad \text { seveloped a policy for obtaining and maintaining consistent access for external personnel despite changes in compliance } \\
\text { - Implemented a secure method of transporting and housing of records with PHI and maintain consistent policy. }\end{array}$ \\
\hline Academic personnel needing clinic access & - Developed a protocol for working with single point of contact in each clinic (e.g., research nurse-coordinators). \\
\hline High clinical coordinator turnover & $\begin{array}{l}\text { - Secured commitment from the centralized clinical system to dedicate coordinator(s) to the project for the duration of the } \\
\text { study period. } \\
\text { - } \quad \text { Conducted biweekly coordinator meetings. } \\
\text { - } \quad \text { Seveloped an online standard operating procedures manual. } \\
\text { ensure data integrity. }\end{array}$ \\
\hline Subject recruitment and retention & $\begin{array}{l}\text { - } \quad \text { Set explicit monthly recruitment goals for each clinic } \\
\text { - } \quad \text { Monitored recruitment via monthly progress reporting and tracking } \\
\text { - } \quad \text { Provided option for passive withdrawal form study } \\
\text { - } \quad \text { Provided option for phone follow-up instead of in-person visit }\end{array}$ \\
\hline Multiple Institutional Review Boards & $\begin{array}{l}\text { - } \quad \text { Established a lead IRB up-front. } \\
\text { - Submitted protocol and addendums to secondary IRBs only after approval is received from lead IRB, as changes may be } \\
\text { mandated by one or both IRBs. } \\
\text { - Maintained a single/same version of currently-approved consent form. } \\
\text { - Required Co-PIs to receive correspondence from both the clinical and academic IRBs. }\end{array}$ \\
\hline
\end{tabular}

\section{Discussion}

The new emphasis on team science and translational studies in real world settings brings opportunities for increasing the positive impacts of science $[22,23]$. However, as the number of partnering organizations increase the challenges of conducting multi-site clinical investigations are often multiplied as seen in this study.

\section{Fostering collaborative communication styles}

As stated previously, a multicenter clinical trial does not fit into a traditional model of hierarchical management, as the clinical team does not manage the academic staff and vice versa. It does however, appear to fit into a traditional model of matrix management, especially if collaborators agree at the beginning to see the co-principal investigators as equal and the coordinators from each entity as also equal. This necessitates the importance of collaboration, rather than direct management, in areas that include communications, IRB roles, and operating procedures.

Over our five-year research study, weekly and later bi-monthly, teleconferences were established for the full research team. Separate coordinator meetings were held in advance of the full team meetings for clarifications on minor recruitment issues and to agree on priority questions for the co-principal investigators. As recommended by Aitken [24], face-to-face biannual retreats were held twice a year and included all team members. This ensured that all team members were able to share information and contribute ideas to assist with continuity for the project.

In their study of the methodological challenges in conducting a multi-site RCT, Kutner et al. identified communication as one the 
three key qualities that characterized successful on-site study teams [4]. Naydeck et al. suggested the use of operational memos, as well as regular conference calls between the coordinating center and other components of a project [3]. Specifically, they emphasized written and verbal communication between on-site study coordinators and their teams, and with their university-based research team. In the present study, biweekly conference calls were employed. Sequential operational memos that detailed standard operating procedures were not utilized, but might have been found useful.

Another communication strategy that could have been used is the establishment of a dedicated "hotline" (or even single point of contact), especially during the recruitment phase, to handle questions from the coordinators at the clinical sites $[5,25]$. Review of questions posed to the hotline at weekly staff meetings could have supported more consistent responses and protocol interpretation across sites. While we did not use this strategy in this study, we recommend it as a means to handle questions expeditiously including allocating appropriate resources for it.

\section{Promoting continuity through team members}

Almost all of our clinical coordinators (13 of the 14) were employed by the healthcare organization and "loaned" to the project for finite periods of time, with hours billed directly to the study. This system did not encourage a sense of ownership of the project and was often confusing to the subjects - as some subjects spoke to a different coordinator for every follow-up visit. Data collection policies were sometimes applied inconsistently due in part to the lack of written operational documentation and, possibly, due to the transient assignments of many of the coordinators. Travel time between the 7 clinics and between appointments placed an additional burden on the coordinators assigned to the more remote clinics.

However, continuity in the clinical coordinator team was established in January 2010, when a new coordinator was assigned to supervise coordinators for 6 of the 7 clinical sites. This included supervising coordinator training, standardizing data collection methods and defining visit flow, developing and implementing coordinator training (and making this a requirement for any coordinators assigned to the study to "help temporarily"), general supervision of coordinators (including regular communication about progress), and quality control. Her leadership allowed the research team to establish clear, performance-oriented recruitment goals and deadlines, and establish consistent policies for communication and compliance between the clinical coordinators. Standardizing protocol training, as recommended by Robb et al. [6] assures that project staff have equivalent training experiences and are equipped to consistently deliver study protocols, regardless of when they join the study team.

\section{Enhancing data access}

In collaborative clinical trials, when employees of academic institutions are responsible for meeting with subjects to collect and record data, lack of access to the EHR can present a challenge. Our study progression was slowed by varying viewpoints on clinical liability (e.g., access to participating subjects' EHR to record vital signs), interinstitutional record keeping (e.g., the issue of maintaining files off-site), and even access to physical facilities (e.g., locked doors for appointments scheduled after 5 p.m.). Frequent and effective communications, instigated by the co-principal investigators, were largely responsible for reducing the potential negative impact of these issues. Ultimately, the coordinator employed by an academic institution was given access to the EHR for patients participating as subjects in this study. Also, establishing a single point of contact at each participating clinic ensured that clinic access was granted expeditiously.

\section{Minimizing staff turnover}

The present study used 14 different coordinators over the 5-year period. Twelve of the 14 , or $86 \%$, were reassigned within the host organization or left employment with the host organization during our study. The continual training and retraining of clinical staff was most costly in the area of "missed follow-up visits" for the designated time points. Coordinator turnover may also have contributed to irregularities in screening strategies and treatment fidelity. For example, some coordinators provided excellent verbal instruction on use of the PDA device, along with the written instructions, while others merely distributed the device and written instructions. The continual turnover of research coordinators, at most of the participating clinics, did not allow for the development of strong "coordinator-subject relationships."

It is important, therefore, to secure a commitment from the healthcare system for dedication of coordinator time for the duration of a study. Developing an online standard operating procedure manual, and implementing policies for online coordinator training and crosstraining, will limit disruptions due to coordinator turnover. Quality assurance procedures, to assess and correct inconsistencies between sites and coordinators, will improve treatment fidelity and maintain data integrity.

\section{Enhancing participant recruitment and retention}

Even without a high degree of coordinator turnover, participant recruitment and retention are generally considered the most challenging aspects of the research process [10-12]. Like many studies, we fell short of our recruitment target (we achieved 376 out of 400 subjects), and did not achieve our targeted 50\% minority recruitment. Subject follow-ups were hindered by the clinical IRB halting study recruitment and followup to determine the best method of record-keeping for the academic institution and how the academic institution could access necessary recruitment criteria from the EHR.

One way we found to improve the retention rate for the study was to offer an option for passive withdrawal, whereby a subject would not be contacted for follow-up surveys, but would still allow their clinical data from the EHR to be used. In this way, they were not required to maintain contact with the investigative team, but continued ongoing contact with their physicians. Allowing subjects to do follow-up surveys by phone, rather than coming into the clinic for an in-person visit, provided a second avenue for increasing retention. However, this increased coordinator load, as vital signs (blood pressure, height, weight) had to be manually culled from the EHR within the designated window for follow-up. It is worth noting that in spite of not meeting our recruitment target and retention, we were able to accomplish major study objectives. For example, we met our study timeline. Nonetheless, future collaborations might want to anticipate untoward consequences regarding time, money or both. Therefore, future studies must consider allocating more time and resources for subject recruitment than we did in this study.

\section{Standardizing IRB policies}

Fully understanding the importance of mandated research policies is a critical factor in conducting successful RCTs. Institutional Review Boards are essential for all types of research and critical to the approval, periodic review, and monitoring of research [26]. Researchers are required to obtain IRB approval before initiating any scientific protocol and funding agencies mandate compliance with the respective IRBs. 
In multi-institutional studies, there may be variations in application formats, as well as review criteria, which can potentially lead to delays in processing and approvals. Gaining approval separately from the IRBs at each of the participating institutions can considerably tax the human resources, in addition to prolonging the whole process by several months. Indeed, we found this to be true.

In the present study, we observed institutional differences in both the application methods (electronic submission versus paper copy) and the level of scrutiny, between the two collaborating organizations - one a healthcare organization and one an academic institution. The clinic used an electronic submission format while paper copies were still employed at the academic health science center. The co-principal investigator, employed by the health science center, was given access to the clinical submission system, but the system was unable to set up a "cc" whereby the co-principal investigator from the academic institution could receive electronic notifications from the clinical IRB.

The negotiation of authority and control between the academic and the clinical IRB respectively, played an important role in our project. Initially, the academic staff took the lead in applying for IRB approval and submitting amendments when required. Once academic IRB approval was received, the clinic staff would submit their requests for approval of identical items. We soon discovered that the clinical IRB more strict required recognition as the lead IRB - in any study where their patients were involved.

Using the clinical IRB as the "lead" IRB reduced the amount of time spent on resubmissions. However, the clinical IRB/compliance staff appeared less aware of the project timeline - even halting subject recruitment twice at critical times in the recruitment process, while compliance staff decided how best to access data from the EHR when an academic institution was involved. Three different record-keeping systems were identified by the clinical compliance staff - each one used then eliminated after a few months - before finally deciding on a workable system. Both institutions accepted CITI training [27] for research on human subjects, with additional HIPAA modules to be completed.

\section{Conclusions}

Qualities characterizing success of multi-site, multi-institutional clinical trials include effective communication between the onsite coordinators, between the clinic and university-based research teams, and between the coordinators and investigators. Of importance also is the commitment by all institutions for advance determination of IRB processes, joint access to records and data management systems, and standardized online training and reporting to eliminate the impact of staff transition and attrition.

\section{Acknowledgements}

The authors wish to gratefully acknowledge the steadfast contributions of Dawn Begaye, Ann Vuong, Omolola Adepoju, Darcy Moudouni, and Phyllis Davis to the study. This work was supported by the National Institutes of Health's National Institute on Minority Health and Health Disparities Program for the Study of Rural and Minority Health Disparities (award number \#1P20MD002295).

\section{Author contributions}

SNF - Conception of study, grant acquisition, study oversight, manuscript preparation, approval of final manuscript.

JWH - Data collection, manuscript preparation, approval of final manuscript.

JNB - Conception of study, grant acquisition, manuscript preparation, approval of final manuscript.

MGO - Conception of study, grant acquisition, manuscript preparation, approval of final manuscript.

\section{References}

1. Richards WD Jr (1988) Network analysis in organizations. In: Ferguson SD and Ferguson S (eds.) Organizational communication. Transaction Books, New Brunswick, New Jersey, pp. 591-621.

2. Hare AP (1992) Group, teams and social interaction: theories and applications Praeger, New York.

3. Naydeck BL, Sutton-Tyrrell K, Burek K, Sopko GS (1996) Organizationa structure and communication strategies of the bypass angioplasty revascularization investigation: a multicenter clinical trial. Control Clin Trials 17: 226-234.

4. Kutner J, Smith M, Mellis K, Felton S, Yamashita T, et al. (2010) Methodological challenges in conducting a multi-site randomized clinical trial of massage therapy in hospice. J Palliat Med 13: 739-744.

5. Sutton-Tyrrell K (1989) Communication strategies for multi-center clinical trials: The BARI hotline. Control Clin Trials 10: 343.

6. Robb SL, Burns DS, Docherty SL, Haase JE (2011) Ensuring treatment fidelity in a multi-site behavioral intervention study: implementing NIH behavior change consortium recommendations in the SMART trial. Psychooncology 20: 11931201.

7. Wilson JF (2006) Health Insurance Portability and Accountability Act Privacy rule causes ongoing concerns among clinicians and researchers. Ann Intern Med 145: 313-316.

8. Shalowitz D, Wendler D (2006) Informed consent for research and authorization under the Health Insurance Portability and Accountability Act Privacy Rule: an integrated approach. Ann Intern Med 144: 685-688.

9. Wolf MS, Bennett CL (2006) Local perspective of the impact of the HIPAA privacy rule on research. Cancer 106: 474-479.

10. Chang BH, Hendricks AM, Slawsky MT, Locastro JS (2004) Patient recruitment to a randomized clinical trial of behavioral therapy for chronic heart failure. BMC Med Res Methodol 4: 8.

11. Ferland D, Fortin PR (1999) Recruitment strategies in superiority trials in SLE: lessons from the study of methotrexate in lupus erythematosus (SMILE). Lupus 8: 606-611.

12. Walson PD (1999) Patient recruitment: US perspective. Pediatrics 104: 619 622.

13. Blanton S, Morris DM, Prettyman MG, McCulloch K, Redmond S, et al. (2006) Lessons learned in participant recruitment and retention: the EXCITE trial. Phys Ther 86: 1520-1533.

14. Abbott L, Grady C (2011) A systematic review of the empirical literature evaluating IRBs: what we know and what we still need to learn. J Empir Res Hum Res Ethics 6: 3-19.

15. Haley WE (2002) A commentary-institutional review board approval and beyond: proactive steps to improve ethics and quality in end-of-life research Gerontologist 42: 109-113.

16. Hanauer SB (2005) Institutional review boards: the cost to academic medical centers. Nat Clin Pract Gastroenterol Hepatol 2: 287.

17. Rushton HG (2008) Institutional review board approval-more red tape or a step in the right direction? J Urol 180: 804-805.

18. Saleem T, Khalid U (2011) Institutional review boards - a mixed blessing. Int Arch Med 4: 19.

19. Stanford Patient Education Research Center, Stanford University School of Medicine, Department of Medicine (2012) Chronic disease self-management program.

20. Forjuoh SN, Bolin JN, Huber JC, Vuong AM, Adepoju OE, et al. (2014) Behavioral and technological interventions targeting glycemic control in a racially/ethnic diverse population: a randomized controlled trial. BMC Public Health 14: 71.

21. Forjuoh SN, Ory MG, Jiang L, Vuong AM, Bolin JN (2014) Impact of chronic disease self-management programs on type 2 diabetes management in primary care. World J Diabetes 5: 407-414.

22. Bennett LM, Gadlin H (2012) Collaboration and team science: from theory to practice. J Investig Med 60: 768-775.

23. http://www.iom.edu/Reports/2013/The-CTSA-Program-at-NIH-Opportunitiesfor-Advancing-Clinical-and-Translational-Research.aspx 
Citation: Forjuoh SN, Helduser JW, Bolin JN, Ory MG (2015) Challenges Associated with Multi-institutional Multi-site Clinical Trial Collaborations: Lessons from a Diabetes Self-Management Interventions Study in Primary Care. J Clin Trials 5: 219. doi:10.4172/2167-0870.1000219

Page 6 of 6

24. Aitken LM, Pelter MM, Carlson B, Marshall AP, Cross R et al. (2008) Effective strategies for implementing a multicenter international clinical trial. J Nurs Scholarsh 40: 101-108.

25. Crow S, Hursh D, Kamons L, Kuntz T, Martin J, et al. (1991) Telecommunications: a tool for information dissemination in multicenter clinical trials. Controlled Clin Trials 12: 675 .
26. Lubowitz JH, Poehling GG, Burkhart SS (2008) Rules of the game: institutiona review boards. Arthroscopy 24: 373-374.

27. CITI Program (2012) Collaborative institutional training initiative at the University of Miami, USA.
Citation: Forjuoh SN, Helduser JW, Bolin JN, Ory MG (2015) Challenges Associated with Multi-institutional Multi-site Clinical Trial Collaborations: Lessons from a Diabetes Self-Management Interventions Study in Primary Care. J Clin Trials 5: 219. doi:10.4172/2167-0870.1000219
Submit your next manuscript and get advantages of OMICS Group submissions

\section{Unique features:}

User friendly/feasible website-translation of your paper to 50 world's leading language Audio Version of published paper

Digital articles to share and explore

\section{Special features:}

400 Open Access Journals

30,000 editorial team

21 days rapid review process

Quality and quick editorial, review and publication processing

Indexing at PubMed (partial), Scopus, EBSCO, Index Copernicus and Google Scholar etc

Sharing Option: Social Networking Enabled

Authors, Reviewers and Editors rewarded with online Scientific Credits

Better discount for your subsequent articles

Submit your manuscript at: http://omicsgroup.org/editorialtracking/clinical-trials 